\title{
Analog RF and mm-Wave Design Tradeoff in UTBB FDSOI: Application to a $35 \mathrm{GHz}$ LNA
}

\author{
Salim El Ghouli ${ }^{1}$, Władysław Grabiński ${ }^{2}$, Jean Michel \\ Sallese $^{3}$ \\ ${ }^{1}$ STMicroelectronics, Crolles, France \\ salim.elghouli@st.com
}

\author{
André Juge ${ }^{1}$, Christophe Lallement ${ }^{4}$ \\ ${ }^{2}$ GMC, Switzerland \\ ${ }^{3}$ EPFL, EDLAB, Lausanne, Switzerland \\ ${ }^{4}$ ICube-University of Strasbourg, Strasbourg, France
}

\begin{abstract}
The state-of-the art RF and millimeter-wave firstcut circuits design requires simple hand calculation methods to avoid time-consuming iterative simulations. The classical MOSFET sizing methods used in advanced technologies, still rely on questionable and inaccurate concepts. Moreover, the pessimistic rules of thumb proposed for older bulk technologies are no more useful and lead to overdesign. This work takes advantage of the Moderate Inversion and uses low and high frequency figures of merit to provide a convenient sizing method for a $35 \mathrm{GHz}$ Low Noise Amplifier (LNA) in $28 \mathrm{~nm}$ UTBB FDSOI technology.
\end{abstract}

Keywords-Transconductance efficiency; gm over Id; Doublegate FETs; FDSOI; UTBB; Analog, RF; Low-Power; Low-Voltage; mm-Wave; LNA

\section{INTRODUCTION}

More and more wireless devices and IoT building blocks rely on CMOS analog and RF circuits to efficiently interact with the physical world. Thanks to the geometry down-scaling, CMOS MOSFETs have been widely used in low-cost and low-power $\mathrm{RF}$ integrated circuits (RFIC). Short-channel related issues, as side effects of the geometry shrink, have been controlled using new architectures and new materials [1][2]. The new architectures allow for excellent electrostatic control [3].

Clearly the UTBB FDSOI technology has proven to be suitable for Analog and RF applications, especially when both power consumption and performance are valued equally. UTBB FDSOI MOSFETs exhibit high analog and RF performances thanks to the reduced parasitic capacitances and resistances [4]. Moreover, the independent back gate provides an interesting degree of freedom that allows tradeoff between power consumption and performance [5][6].

Several circuit-topology-based techniques such as current reuse are proposed in analog design to optimize performance and power consumption [7]. These techniques are out of this paper scope. In this work, the UTBB FDSOI characteristics are used for first-cut analog sizing method. In particular, the transconductance efficiency versus inversion coefficient (IC) studied in [6] is used to determine the width of the MOSFET while the transit frequency $\left(\mathrm{f}_{\mathrm{T}}\right)$ versus IC charts are used for length selection. The design method, using relaxed length and Moderate Inversion (MI) regime, provides a valuable tradeoff between gain, power consumption and performance while MOSFET width is kept reasonably large and short channel effects are mitigated. An eye is also kept on passive devices limitations from the very beginning of the sizing flow.

This paper is organized as follows. First, the classical analog design methods, based on inherited rules of thumb, are briefly discussed in Section II. Second, the UTBB FDSOI technology capabilities for Analog and RF applications are reminded in Section III. Third, the transconductance $\left(\mathrm{g}_{\mathrm{m}}\right)$ over drain current ( $\left.\mathrm{I}_{\mathrm{D}}\right)$ based design method is presented in Section IV. Fourth, the high frequency performance versus IC and MOSFET length is assessed in Section V. Finally, a $35 \mathrm{GHz}$ LNA design tradeoff in Moderate Inversion is proposed in Section VI and a conclusion is given in Section VII.

\section{CLASSICAL DESIGN SIZING METHODS IN ANALOG AND RF}

Sizing MOSFETs through iterative simulations and trial and error practices takes considerable amount of the precious design time. Optimum design tradeoff is hardly achieved using these methods [8]. Furthermore, hand calculation based analog design remains the method of choice to minimize iterative simulations and enables intuitive design. The classical hand calculation sizing methods are still based on inaccurate and questionable concepts such as the gate voltage overdrive $\left(\mathrm{V}_{\mathrm{ov}}=\mathrm{V}_{\mathrm{GS}}-\mathrm{V}_{\mathrm{TH}}\right.$, where $\mathrm{V}_{\mathrm{TH}}$ is the threshold voltage), and pessimistic rules of thumb such as the shortest possible length for higher $\mathrm{f}_{\mathrm{T}}$, higher $\mathrm{V}_{\mathrm{ov}}$ constraint, and a maximum operation frequency of $\mathrm{f}_{\mathrm{T}} / 10$.

Design in Moderate Inversion has become attractive in advanced technologies as it offers the optimum trade-off between speed, transconductance, and power consumption [9][10]. However, the classical gate voltage overdrive is becoming a poor metric for MOSFET inversion level assessment in advanced technologies as it is based on conflicting definitions of the threshold voltage [11]. The latter is defined as the $\mathrm{V}_{\mathrm{GS}}$ value at the onset of the Strong Inversion (SI) and extracted using several methods and criteria leading to uncorrelated definitions. Moreover, gate overdrive voltage can only be used for the hand calculation sizing method based on SI square law that miserably fails to predict $g_{m} / I_{D}$ in MI as seen in Fig. 1. Even for a long channel device, the error is more than $100 \%$ at $\mathrm{V}_{\mathrm{ov}}=100 \mathrm{mV}$. For short channel devices (e.g. $\mathrm{L}=30$ $\mathrm{nm}$ in Fig. 1), the simple square law is not valid in all levels of inversion. Moreover, for a double gate transistor such as the 


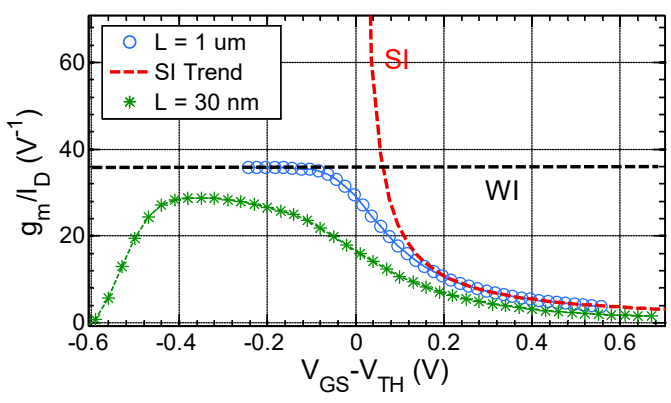

Fig. 1 Square law model (SI) and weak inversion exponential model failures to predict measured $\mathrm{g}_{\mathrm{m}} / \mathrm{I}_{\mathrm{D}}$ in $\mathrm{MI}$ for a long and short channel devices. $\mathrm{V}_{\mathrm{TH}}$ is defined here as the $\mathrm{V}_{\mathrm{GS}}$ at the maximum of $\partial \mathrm{C}_{\mathrm{gd}} / \partial \mathrm{V}_{\mathrm{GS}}$.

UTBB FDSOI MOSFET, we experimentally observe two separate threshold voltages in the forward back gate bias condition while considering the $\mathrm{C}-\mathrm{V}$ and its derivative both shown respectively in Fig. 2 and its Inset. Consequently, the gate overdrive voltage becomes impractical for describing the level of inversion of the advanced MOSFET architectures. It should be noted that $\mathrm{V}_{\text {ov }}$ can become negative in MI and for lower levels of inversion, and thus becomes useless.

In RF circuits, designers tend to use shortest devices to get highest possible transit frequency $\mathrm{f}_{\mathrm{T}}$ and consequently better performance. However, shortest MOSFETs are subject to detrimental short channel effects, lower intrinsic voltage gain, and higher mismatch. In Fig. 3, Drain-Induced-BarrierLowering (DIBL) versus the gate length is shown for bulk and FDSOI technologies. Using relaxed and non-minimal lengths provides lower DIBL effect and lower variability while frequency performance is still high enough for the majority of today's applications in the RF spectrum as shown in Erreur ! Source du renvoi introuvable. where $\mathrm{f}_{\mathrm{T}}$ is plotted versus the Inversion Coefficient (IC) for various MOSFET lengths in 28 nm UTBB FDSOI technology. IC is defined as:

$$
I C=\frac{I_{D}}{I_{\square} \cdot\left(\frac{W}{L}\right)}
$$

where $\mathrm{I}_{\mathrm{D}}, \mathrm{W}$ and $\mathrm{L}$ are respectively the drain current, the width, and the length. I $\square$ is the square current at the middle of the moderate inversion with a value of about $0.7 \mu \mathrm{A}$ for $28 \mathrm{~nm}$ UTBB FDSOI [6]. For $\mathrm{L}=100 \mathrm{~nm}$, the maximum transit frequency is still in the mm-Wave spectrum $(>100 \mathrm{GHz})$ for the FDSOI technology while DIBL is reduced by $23 \mathrm{mV} / \mathrm{V}$ with respect to $\mathrm{L}=28 \mathrm{~nm}$. Moreover, according to (1) and for fixed $\mathrm{I}_{\mathrm{D}}$ and inversion level IC, the device area increases as $\mathrm{L}^{2}$, which is beneficial for local mismatch effect.

Normalized module and phase of the transadmittance $Y_{21}$ are respectively shown in Erreur ! Source du renvoi introuvable. and Erreur ! Source du renvoi introuvable. versus frequency for different lengths and at $\mathrm{V}_{\mathrm{GS}}=0.5 \mathrm{~V}$. The transadmittance is normalized using $\mathrm{I}_{\square} / \mathrm{U}_{\mathrm{T}}$ where $\mathrm{U}_{\mathrm{T}}$ is the thermal voltage. All devices are operating in the MI regime. At $\mathrm{V}_{\mathrm{GS}}=0.5 \mathrm{~V}$, shorter channels $(\mathrm{L}<100 \mathrm{~nm})$ are biased at an inversion coefficient of 6.3, while for $\mathrm{L}=100 \mathrm{~nm}, \mathrm{IC}=5.1$. For $\mathrm{L}=100 \mathrm{~nm}$ and at $\mathrm{IC}=$ 5.1 , with a transit frequency $\mathrm{f}_{\mathrm{T}}$ of $70 \mathrm{GHz}$, the $\mathrm{f}_{\mathrm{T}} / 10$ rule of thumb gives, $7 \mathrm{GHz}$. At this limit frequency no degradation on the $\mathrm{Y}_{21}$ module is observed and a phase shift of less than $4^{\circ}$ is

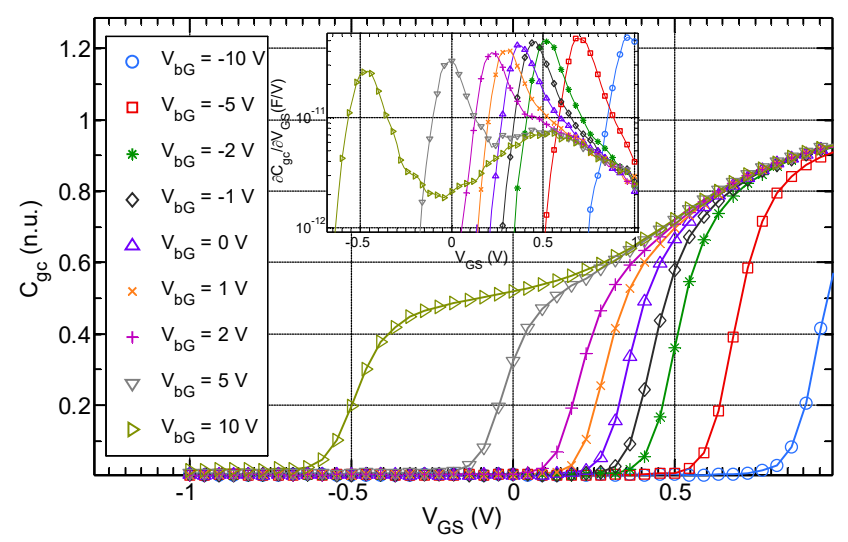

Fig. 2 Measured gate to channel capacitance normalized using front oxide capacitance $\left(\mathrm{C}_{\mathrm{ox}}\right.$.W.L) with respect to $\mathrm{V}_{\mathrm{GS}}$ for various $\mathrm{V}_{\mathrm{bG}}$ and its derivative (Inset) for N-type UTBB FDSOI MOSFET.

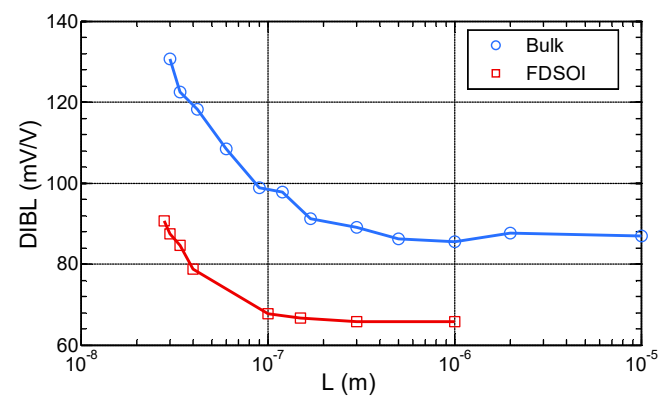

Fig. 3 Comparison of DIBL between FDSOI and Bulk in saturation $\left(\mathrm{V}_{\mathrm{DS}}=1 \mathrm{~V}\right)$ and $\mathrm{V}_{\mathrm{bG}}=0 \mathrm{~V}$.

measured. The finger length of the measured structures is $1 \mu \mathrm{m}$ and the lateral gate distributed effect is largely contributing to the measured phase shift. Though, a phase shift of $10^{\circ}$ is measured at $19 \mathrm{GHz}$ with no degradation on $\left|\mathrm{Y}_{21}\right|$. In summary, it is clearly evidenced that non minimal channel lengths (i.e. $\mathrm{L}>$ $30 \mathrm{~nm}$ in $28 \mathrm{~nm}$ node) in advanced technologies provide high enough $\mathrm{f}_{\mathrm{T}}$ values that can still be used to design RF circuits, while the conservative $f_{T} / 10$ rule of thumb is misleading, in particular at low inversion levels. 'Common' rules are then inaccurate and lead to overdesign. To overcome these issues, optimal geometries and bias conditions are approximated using time-consuming iterative numerical simulations. Other sizing methods based on the Inversion Coefficient concept have been proposed [12]. However, in many circuits where passive elements play a key role such as Low Noise Amplifier (LNA), circuits are optimized using time-consuming iterations of the IC based method since no passive part related constraints are considered in the active part optimization [13].

\section{ADVANTAGES OF UTBB FDSOI TECHNOLOGY}

In the UTBB FDSOI technology, MOSFET channel is formed in a thin silicon film separated from the substrate by an oxide film called the Buried OXide (BOX). In $28 \mathrm{~nm}$ FDSOI technology, the final silicon film is $7 \mathrm{~nm}$ thick after process [2]. This architecture provides with multiple advantages for high performance and low power applications. In the addition of the well-known SOI technology advantages [14][15], UTBB 


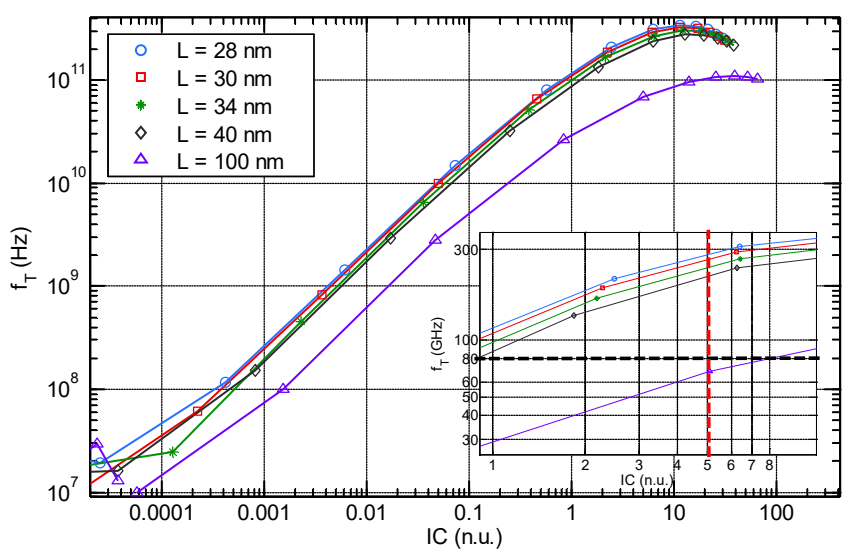

Fig. 4 Measured $\mathrm{f}_{\mathrm{T}}$ versus IC for various MOSFET lengths in saturation $\left(\mathrm{V}_{\mathrm{DS}}=1 \mathrm{~V}\right)$. Inset gives a focus on the second part of the MI $(1<\mathrm{IC}<10)$

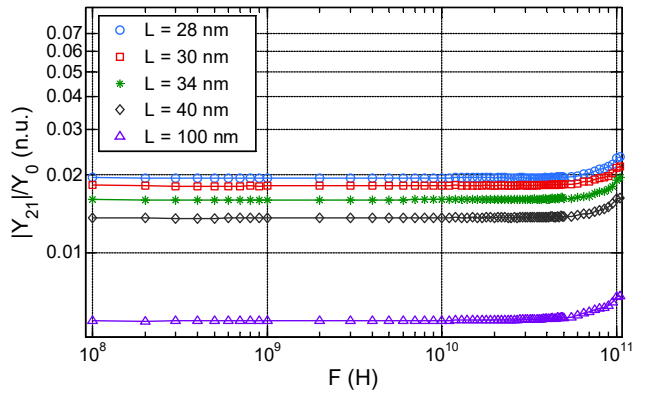

Fig. 5 Measured and normalized $\left|Y_{21}\right|$ versus frequency for various lengths and finger length is $1 \mu \mathrm{m}$.

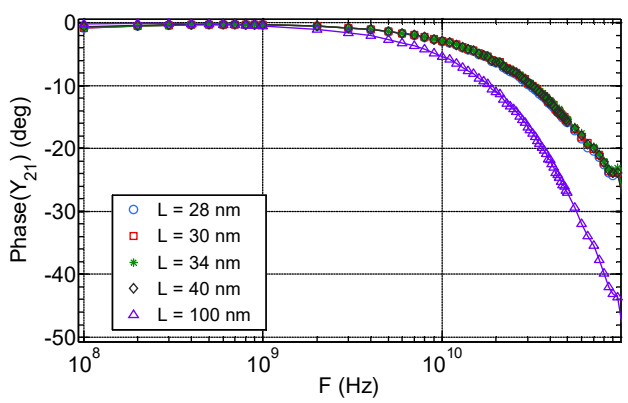

Fig. 6 Phase shift of the transadmittance $Y_{21}$ versus frequency for various lengths and finger width $\mathrm{W}_{\mathrm{f}}=1 \mu \mathrm{m}$.

FDSOI technology features lower parasitic capacitances and then high-speed operation. The harmful parasitic substrate coupling is avoided in the UTBB FDSOI by introducing the Ground Plane (GP) which is a highly doped region underneath the thin BOX [16]. The ground-plane implantation under the BOX is well-type in the structures studied in this work. FDSOI technology allows co-integration of both bulk and SOI devices on the same die thanks to BOX opening for the bulk parts with a dedicated mask [17].

With less parasitic capacitances, supply voltage can be lowered for reduced power consumption with still high speed operation. Other advantages of UTBB FDSOI are steep subthreshold slope [8], reduced SCE (cf. Fig. 3), tolerance to radiation as for standard SOI and high temperatures, even though buried oxide isolation is known to give birth to temperature increase because of self-heating effect [17]. However, thanks to a thinner BOX in the UTBB FDSOI, thermal effects influence on device parameters are limited in comparison with standard SOI [18].

The advantages of the UTBB FDSOI technology make it possible to implement high performance MOSFETs operating at a low voltage, specifically in the moderate inversion regime. An understanding of the fundamental behavior of the UTBB FDSOI MOSFETs at high frequency is essential for circuit design and a sizing method in MI is a must.

\section{IV. $\mathrm{G}_{\mathrm{M}}$ OVER I INVARIANCE BASED METHOD}

In digital CMOS circuits, static power consumption is mainly related to the leakage current. However, in analog circuits, biasing current is the main contributor for circuit power consumption. Thus, the devices used in analog blocks need to be permanently biased in the appropriate region. Besides power supply voltage lowering in recent technology nodes, the current budget should be reduced as well.

In RF circuits, a good tradeoff between speed and low current budget is satisfied in moderate inversion. However, for advanced devices such as the asymmetric double gate MOSFETs, the validity of classical hand calculation expressions is questionable. The lack for simple expressions for hand calculation can be reasonably contained using measurement based charts. The measured transconductance efficiency charts assessed in [6], can be used to size the transistor and to ensure its operation in moderate inversion. The invariance of the $g_{m}$ over $I_{D}$ chart in $M I$ for $L \geq 100 \mathrm{~nm}$ makes is easy to generate the required chart. If we take into account the slope degradation using the slope factor $\mathrm{n}_{1}$, shorter geometries can also be accounted for with same merged chart. In Erreur! Source du renvoi introuvable., $g_{m}$ over $I_{D}$ charts are shown versus $I C$. A longer geometry $(\mathrm{L}=1 \mu \mathrm{m})$ is also shown for comparison. Using these charts, the $g_{m}$ over $I_{D}$ value can be reasonably retrieved for any selected IC and for each displayed geometry from WI to SI.

Recently, it is claimed that preselecting drain current $\left(\mathrm{I}_{\mathrm{D}}\right)$, IC, and channel length is the most efficient way to size MOSFETs in analog circuits [12]. However, the proposed simple expressions of MOS performance mainly rely on EKV formalism which has been extrapolated to a symmetric DG in [18] but not yet transposed to the asymmetric DG. However, the choice of the inversion coefficient as a measure of the inversion level in the channel happens to be totally justified since it accurately describes the inversion charge and consequently the operating regime for single or double gate MOSFETs [12][6]. According to Erreur! Source du renvoi introuvable., selecting an IC value is equivalent to setting $g_{m}$ over $I_{D}$ of the device. Moreover, based on (1), once IC an $I_{D}$ are known, the geometry ratio $(\mathrm{W} / \mathrm{L})$ can be calculated.

\section{HIGH FREQUENCY PERFORMANCE ASSESSMENT}

One of the interesting high frequency FoM is the transit frequency $\mathrm{f}_{\mathrm{T}}$ that estimates the high frequency amplification limit and also provides an insight of the transconductance to input capacitance ratio $\left(\mathrm{f}_{\mathrm{T}} \approx \mathrm{g}_{\mathrm{m}} / \mathrm{C}_{\mathrm{gg}}\right.$ ) of the MOSFET. The transit frequency versus IC charts for several lengths provide 


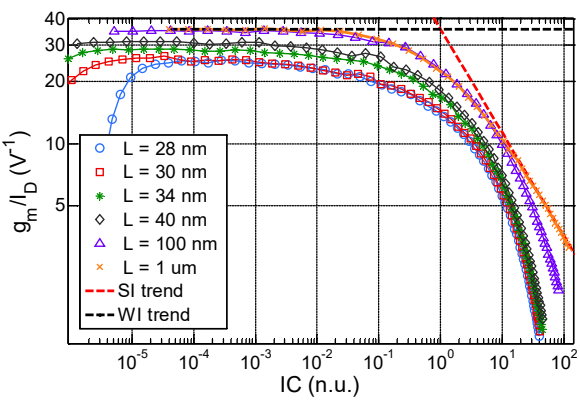

Fig. $7 g_{m}$ over $I_{D}$ charts versus IC for several short NMOS along with a longer channel $(\mathrm{L}=1 \mu \mathrm{m})$ for comparison.

high frequency performance limits since $\mathrm{f}_{\mathrm{T}}$ represents the frequency at which current gain falls to unity. However, it should be noted that the transistor can operate at any frequency even beyond this limit, provided that non-quasi-static related limitations are carefully taken into account and modeled. The module and phase of the transadmittance versus frequency charts (e.g. Erreur! Source du renvoi introuvable. and Erreur ! Source du renvoi introuvable.) can also be used to estimate the phase shift and module drop when frequency is set beyond $\mathrm{f}_{\mathrm{T}}$. As stated in Section II, the rule of thumb stating that maximum frequency of operation must be lower than $\mathrm{f}_{\mathrm{T}} / 10$ is too stringent. This rule of thumb finds its origin in the validity of the quasi-static model proposed in [19] and thus is essentially a misinterpretation.

\section{LNA DESIGN USING MI TRADEOFF IN UTBB FDSOI}

In this Section, the focus will be on another challenge which is the transistor sizing. The RF designer dilemma is to calculate transistor geometry and current in order to get maximum amplification, minimum degradation of the signal-to-noise ratio, and minimum power consumption. The goal is to provide an illustration of a design method mainly based on the charts and properties described in Section IV and V.

One of the key building blocks in a wireless system is a Low Noise Amplifier (LNA). The LNA circuit amplifies the input signal with minimum degradation of the signal-to-noise ratio. One of the challenges RF designers must face is the simultaneous matching of the noise and the input impedance for the same source impedance. This challenge is beyond the scope of this paper therefore ideal impedance matching is considered here. However, a constraint on the value of the inductors is considered in order to account for integrated inductors with acceptable quality factors.

One of the most used circuit topologies for an LNA is the narrowband cascode. The cascode transistor is used to isolate the input from the inductive load. In particular, the Miller effect is reduced for the input transistor. However, using a cascode topology leads to a minimal room for drain to source voltage required to set both transistors in saturation. This also corroborates the need for MI operation as $\mathrm{V}_{\mathrm{DSAT}}$ is lower in this regime. The circuit of the cascode LNA is shown in Fig. 8. The transistor $\mathrm{M}_{1}$ is the input transistor and $\mathrm{M}_{2}$ is the cascode transistor. Both transistors are chosen to have same geometry. Degeneration inductor $\mathrm{L}_{\mathrm{s}}$ and input series inductor $\mathrm{L}_{\mathrm{g}}$ are used for input impedance matching.

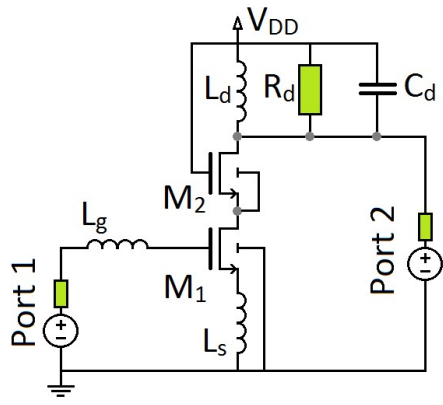

Fig. 8 LNA cascode circuit.

The following specifications are taken as an example: Drain current is $2 \mathrm{~mA}$, power gain is greater than or equal to 8 , operation frequency is $\mathrm{F}_{0}=35 \mathrm{GHz}$, minimal noise figure NFmin is no more than $2 \mathrm{~dB}$, and input impedance is matched for $50 \mathrm{ohm}$. In order to size the circuit components and meet the aforementioned specifications, the following 3 steps are followed:

\section{A. IC selection}

The maximum value of the FoM given in (2) and representing the trade-off between high frequency performance, power consumption, and noise figure is located in the second half of the MI for short channels as shown in Erreur ! Source du renvoi introuvable. for two channel lengths $(\mathrm{L}=30 \mathrm{~nm}$ and $1 \mu \mathrm{m})$.

$$
F O M=\frac{g_{m}}{I_{D}} \cdot \frac{f_{T}}{N F_{\text {min }}}
$$

Optimal IC lays, at least theoretically, between IC $=4$ and $\mathrm{IC}=10$. Depending of the length selection, corresponding IC will be chosen where FOM is maximum.

\section{B. Passives related constraints and Length selection}

In order to avoid passive components with degraded quality factors Q, some constraints are considered prior to MOSFETs sizing. The values are chosen within the integrated $\mathrm{mm}$-Wave inductor values $[25 \mathrm{pH}-100 \mathrm{pH}]$ as in [20]. The real part of the LNA input impedance is mainly tuned using degeneration inductor $\mathrm{L}_{\mathrm{s}}$. The expression of this real part is given in (3), provided that $M_{1}$ intrinsic conductance influence on input impedance is neglected:

$$
\operatorname{Re}\left[Z_{\text {in }}\right]=\frac{g_{m} L_{s}}{C_{g s}} \approx 2 \pi f_{T i} L_{s}
$$

where $\mathrm{C}_{\mathrm{gs}}$, and $\mathrm{f}_{\mathrm{Ti}}$ are the gate-source, and intrinsic transit frequency of $M_{1}$, respectively. The specification related to impedance matching fixes the real part of $Z_{\text {in }}$ at $50 \mathrm{ohm}$ and consequently a relation between the inductance $\mathrm{L}_{\mathrm{s}}$ and the transit frequency is given by (3). If we consider a maximum $\mathrm{L}_{\mathrm{s}}$ inductance of $100 \mathrm{pH}, \mathrm{M}_{1}$ transit frequency $\mathrm{f}_{\mathrm{Ti}}$ should be greater than $80 \mathrm{GHz}$. According to Inset of Erreur ! Source du renvoi introuvable., for operation at $\mathrm{IC}=4$, the MOSFET length that guarantees the required $\mathrm{f}_{\mathrm{T}}$ is strictly greater than $\mathrm{L}=100 \mathrm{~nm}$. To obtain acceptable noise figures and using $\mathrm{NF}_{\text {min }}$ versus IC charts (not shown here), length should also be lower than $100 \mathrm{~nm}$. In order to verify the noise figure specification and inductor value 
constraint, we will consider two channel lengths that are $\mathrm{L}=40$ $\mathrm{nm}$ and $\mathrm{L}=70 \mathrm{~nm}$, with

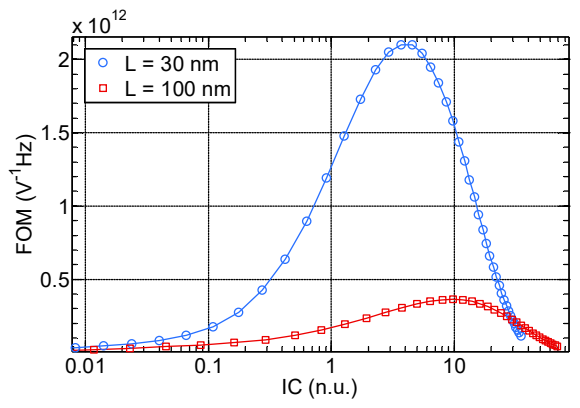

Fig. 9 FOM versus IC for two lengths.

FOM maximizing respectively at $\mathrm{IC}=5$ and $\mathrm{IC}=9$. Both geometries provide acceptable high frequency performance. Moreover, $\mathrm{L}=40 \mathrm{~nm}$ and $\mathrm{L}=70 \mathrm{~nm}$ MOSFETs are less subject to DIBL and Mismatch than minimal length. Thus, an $\mathrm{L}_{\mathrm{s}}$ value of $33 \mathrm{pH}$ and $39 \mathrm{pH}$ for respectively $\mathrm{L}=40 \mathrm{~nm}$, and $\mathrm{L}=70 \mathrm{~nm}$ MOSFET based LNAs are found.

The LNA is designed to operate at $\mathrm{F}_{0}=35 \mathrm{GHz}$ and consequently, to cancel the input impedance imaginary part at this resonance frequency, the inductor $\mathrm{L}_{\mathrm{g}}$ is tuned with a target value given by [21]:

$$
L_{g}=\frac{1}{\left(2 \pi F_{0}\right)^{2} \cdot C_{i n}}-L_{s}
$$

where $C_{i n}$ is including the total $M_{1}$ gate capacitance $C_{g g}$ and the Miller effect of the gate-drain capacitance $\left(\mathrm{C}_{\mathrm{gd}}\right)$.

\section{Width and $V_{G S}$ calculations}

The Width is calculated using the following expression:

$$
W=\frac{I_{D}}{I C \cdot I_{\square}} \cdot L
$$

Close width values of $22.8 \mu \mathrm{m}$ and $22.1 \mu \mathrm{m}$ are found for respectively $\mathrm{L}=40 \mathrm{~nm}$ and $\mathrm{L}=70 \mathrm{~nm}$ based LNA circuits.

The gate voltage overdrive with respect to IC charts (not shown here) are used to retrieve the gate to source voltage corresponding to the two IC values for each length. The calculated $\mathrm{V}_{\mathrm{GS}}$ values are respectively $0.49 \mathrm{~V}$ and $0.55 \mathrm{~V}$.

The results of the above sizing are shown in Fig. 10 versus frequency and summarized in Table 1 for the operation frequency $\mathrm{F}_{0}=35 \mathrm{GHz}$.

Table 1 Summary of the two LNAs parameters at $\mathrm{F}_{0}=35 \mathrm{GHz}$.

\begin{tabular}{|c|c|c|c|}
\hline & $\mathrm{S}_{21}$ & $\mathrm{~S}_{11}$ & NFmin \\
\hline LNA $(\mathrm{L}=40 \mathrm{~nm})$ & 10.9 & -31 & 1.5 \\
\hline LNA $(\mathrm{L}=70 \mathrm{~nm})$ & 8.6 & -21.5 & 2 \\
\hline
\end{tabular}

Power gain is lower for the $\mathrm{L}=70 \mathrm{~nm}$ based LNA because of the higher IC and consequently lower transconductance efficiency. The input impedance matching is acceptable for both LNA circuits. The NFmin value is higher for the longer channel based LNA as expected, however this can be lowered using optimized layout. The power consumption of both LNAs is 2 $\mathrm{mW}$ which is excellent for low-power applications.

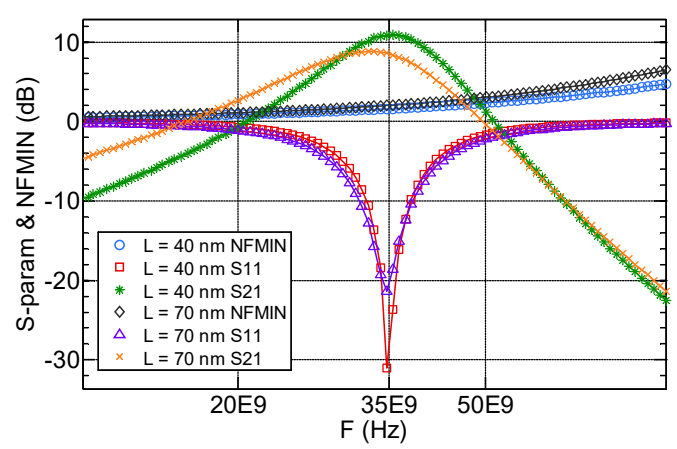

Fig. 10 Power gain S21, Input Match S11, and minimal noise figure NFmin with respect to frequency for two LNA circuits (first with MOSFET length of $40 \mathrm{~nm}$ and second with $70 \mathrm{~nm}$ ).

\section{CONCLUSION}

In this work, a constrained sizing methodology is proposed for the UTBB FDSOI MOSFET. The methodology is based on $\mathrm{f}_{\mathrm{T}}$ versus IC measured or simulated curves for the length selection, and on $g_{m}$ over $I_{D}$ charts for the width calculation. The Inversion Coefficient is fixed to a value where the MOSFET optimum performance is expected, making this analysis dependent only on the channel current, and 'independent' of the front and back gate biases, a great simplification in terms of analysis. The methodology takes into account the passive components limitations and thus requires less if not minimal iterations.

\section{REFERENCES}

[1] D. Hisamoto, T. Kaga, Y. Kawamoto, and E. Takeda, "A fully depleted lean-channel transistor (DELTA)-a novel vertical ultra-thin SOI MOSFET," in International Technical Digest on Electron Devices Meeting, 1989, pp. 833-836.

[2] N. Planes et al., "28nm FDSOI technology platform for high-speed lowvoltage digital applications," in VLSI Technology (VLSIT), 2012 Symposium on, 2012, pp. 133-134.

[3] F. Balestra, S. Cristoloveanu, M. Benachir, J. Brini, and T. Elewa, "Double-gate silicon-on-insulator transistor with volume inversion: A new device with greatly enhanced performance," IEEE Electron Device Lett., vol. 8, no. 9, pp. 410-412, Sep. 1987.

[4] J.-P. Raskin, "FinFET versus UTBB SOI - a RF perspective," in Solid State Device Research Conference (ESSDERC), 2015 45th European, 2015, pp. 84-88.

[5] J.-P. Noel et al., "Multi-VT UTBB FDSOI Device Architectures for LowPower CMOS Circuit," IEEE Trans. Electron Devices, vol. 58, no. 8, pp. 2473-2482, Aug. 2011.

[6] S. E. Ghouli et al., "Experimental gm/ID Invariance Assessment for Asymmetric Double-Gate FDSOI MOSFET," IEEE Trans. Electron Devices, vol. 65, no. 1, pp. 11-18, Jan. 2018.

[7] A. N. Karanicolas, "A 2.7-V 900-MHz CMOS LNA and mixer," IEEE J. Solid-State Circuits, vol. 31, no. 12, pp. 1939-1944, Dec. 1996.

[8] D. Foty, M. Bucher, and D. Binkley, "Re-interpreting the MOS transistor via the inversion coefficient and the continuum of gms/Id," in 9th International Conference on Electronics, Circuits and Systems, 2002, 2002, vol. 3, pp. 1179-1182 vol.3.

[9] A. Shameli and P. Heydari, "Ultra-low power RFIC design using moderately inverted MOSFETs: an analytical/experimental study," in 2006 IEEE Radio Frequency Integrated Circuits (RFIC) Symposium, 2006, p. 4 pp.-.

[10] S. E. Ghouli et al., "Analog and RF modeling of FDSOI UTBB MOSFET using Leti-UTSOI model," in 2016 MIXDES - 23rd International 
Conference Mixed Design of Integrated Circuits and Systems, 2016, pp. 41-46.

[11] A. Ortiz-Conde, F. J. García-Sánchez, J. Muci, A. Terán Barrios, J. J. Liou, and C.-S. Ho, "Revisiting MOSFET threshold voltage extraction methods," Microelectron. Reliab., vol. 53, no. 1, pp. 90-104, Jan. 2013.

[12] D. M. Binkley, "Tradeoffs and Optimization in Analog CMOS Design," in 14th International Conference on Mixed Design of Integrated Circuits and Systems, 2007. MIXDES '07, 2007, pp. 47-60.

[13] F. Fadhuile, T. Taris, Y. Deval, C. Enz, and D. Belot, "Design methodology for low power RF LNA based on the figure of merit and the inversion coefficient," in 2014 21st IEEE International Conference on Electronics, Circuits and Systems (ICECS), 2014, pp. 478-481.

[14] A. O. Adan, T. Naka, A. Kagisawa, and H. Shimizu, "SOI as a mainstream IC technology," in 1998 IEEE International SOI Conference Proceedings (Cat No.98CH36199), 1998, pp. 9-12.

[15] S. Cristoloveanu and F. Balestra, "SOI technologies, materials and devices," in Semiconductor Conference, 1996., International, 1996, vol. 1, pp. 3-12 vol.1.
[16] C. Fenouillet-Beranger et al., "FDSOI devices with thin BOX and ground plane integration for $32 \mathrm{~nm}$ node and below," Solid-State Electron., vol. 53, no. 7, pp. 730-734, 2009.

[17] C. Fenouillet-Beranger et al., "Hybrid FDSOI/bulk High-k/metal gate platform for low power (LP) multimedia technology," in 2009 IEEE International Electron Devices Meeting (IEDM), 2009, pp. 1-4.

[18] J.-M. Sallese, F. Krummenacher, F. Prégaldiny, C. Lallement, A. Roy, and C. Enz, "A design oriented charge-based current model for symmetric DG MOSFET and its correlation with the EKV formalism," Solid-State Electron., vol. 49, no. 3, pp. 485-489, Mar. 2005.

[19] Y. Tsividis, Operation and modeling of the MOS transistor, 2nd ed. New York: Oxford University Press, 1999.

[20] J. Brinkhoff, K. S. S. Koh, K. Kang, and F. Lin, "Scalable Transmission Line and Inductor Models for CMOS Millimeter-Wave Design," IEEE Trans. Microw. Theory Tech., vol. 56, no. 12, pp. 2954-2962, Dec. 2008.

[21] B. Razavi, RF Microelectronics. Prentice Hall, 2012. 